\title{
An insight into the pre-operative experiences of Ghanaian general surgical patients
}

\author{
Aziato L, Adejumo O.
}

\begin{abstract}
The unknown outcome of surgery has always been a source of anxiety for patients and their relatives. However, the experiences of Ghanaian surgical patients have not been adequately explored. This study sought to have an in-depth exploration of the pre- operative experiences of Ghanaian general surgical patients to inform effective pre- operative care. The study employed an ethnographic design and was conducted at two hospitals in Accra. Thirteen general surgical patients were purposively recruited and interviewed. Data analysis occurred concurrently and themes that emerged included reaction to impending surgery with sub-themes of inappropriate disclosure, fear of death, readiness for surgery, and effect of waiting in the theatre. Also, the theme information gap had sub-themes of pre-operative care, expectations at the theatre, and undue delays. The study emphasized the need for health professionals to provide effective education to the public and patients, on surgery and its effects, to curb negative perceptions about surgery.
\end{abstract}

\section{Key words}

Pre-operative care, pre-surgery experience, general surgery, emergency surgery, planned surgery, ethnography

Pre-operative care, rendered to a patient before surgery is performed, is an important aspect of peri-operative experience. The pre-operative period is characterised by anxiety attributed to fear of the unknown, pain and death (Rosen, Svensson, \& Nilsson, 2008). However, the Ghanaian surgical patients' pre-operative experiences have not been fully explored. An insight into the pre-operative experiences of surgical patients could inform the future delivery of care. This could subsequently enhance the post-operative management and recovery to these patients. An effective pre-operative care manual would ensure early recovery and early discharge of surgical patients.

\section{Background}

The surgical experience comes with the probability of complications and these create fear and anxiety for surgical patients. Historically, surgery was much feared before the advent of Diethyl ether ini.846 which prevented pain during surgery. Surgery was a feared 
experience because of its' associated pain, infection, and bleeding. However, technological advancement over the years has rendered surgery safer with more successful outcomes. It is reported that while some surgical patients have lost their lives during surgery, these incidences are dwindling in recent years (Badoe, 2009). Thus, skilled personnel and sophisticated equipment have contributed to safe surgery in contemporary health care. However, literature continues to report an inadequately managed post-operative pain (Clegg-Lamptey \& Hodasi, 2005; Qu, Sherwood, McNeill, \& Zheng, 2008). Also, literature reports persistence of surgical wound infection (Stepanovic et al., 2007). It pre-supposes that patients scheduled for surgery could experience any of these problems after surgery and the exhibition of worry or anxiety pre-operatively may continue to dominate the surgical context.

Pre-operative care varies in scope and thoroughness depending on the age, type and urgency of surgery, existing disease condition, protocol of unit/hospital, facilities available, and among others. Thus, pre-operative care for planned surgeries involves a multidisciplinary team of experts depending on the patients' condition such as the nurse, the anaesthetists, surgeon, psychologists, and radiologists and among others. It is recognized that anaesthetists have a major responsibility in assessing pre-operative patients to determine the suitable anaesthesia to give. The literature finds it mandatory for all pre-operative patients to be assessed before anaesthesia is administered and the assessment helps to forestall complications intra-operatively or post-operatively (Matthias \& Samarasekera, 2012; Solca, 2006).

The pre-operative care also involves psychological care. The patient and relatives are provided with information about the surgical procedure and they are allowed to ask questions that help to make the appropriate decisions about the impending surgery and subsequent care (Mullen, van den Borne, \& Breemhaar, 2000). Some previous studies have realized that adequate provision of information before surgery reduces pre-operative anxiety and also enhances post-operative recovery (Guo, East, \& Arthur, 2012). However, other authors also found no significant effect of pre-operative information and reduction in anxiety (Asilioglu \& Celik, 2004).

Pre-operative physiological care involves radiological and laboratory investigations as well as other assessments. The surgical team is guided by the physiological variables obtained and appropriate history to ensure that appropriate clinical decisions are made. When the patient is declared fit for surgery, the nurse prepares the patient physically for the surgery by removing items contra-indicated for surgery and also shaves the patient as necessary. The literature has also examined pre-operative fasting and it is realized that sometimes surgical patients are starved unduly. It is observed that with modern technological advances in anaesthesia and surgery, many hours of pre-operative starving may not be necessary (Brady, Kinn, Stuart, \& Ness, 2010; Soreide \& Ljungqvist, 2006).

In the Ghanaian clinical context, surgical patients undergoing planned surgeries are required to have an assessment at the anaesthetists' clinic where patients are informed about the impending surgery, what is expected of them, and their laboratory results are also assessed for any abnormalities. Patients found to have problems are referred to the doctors for management. There is no other formal pre-operative education or information facility or 
manual within the health system for patients and their relatives. Also, patients undergoing emergency surgeries do not experience the anaesthetists' clinic and the scope of pre-operative assessment provided depends on the urgency of the impending surgery.

Previous studies explored the anxiety levels of pre-operative patients in other countries (Guo et al., 2012) and the impact of pre-operative information among specific surgical procedures such as orthopaedics, cardiac surgery, and bariatric surgery (Sjoling, Nordahl, Olofsson, \& Asplund, 2003). No study was retrieved on the Ghanaian surgical patients' pre-operative experience. It pre-supposes that the pre-operative issues specific to the Ghanaian context may not have been adequately explored.

\section{Purpose of Study}

The purpose of this article is to describe the pre-operative experiences of surgical patients in selected hospital settings in Ghana.

\section{Methodology}

\section{Design}

The study adopted a qualitative approach by applying the principles of focused ethnography. Ethnographic principles help in the study of a particular context or culture such as the surgical environment and the full understanding of a phenomenon as applied by previous researchers (Arber, 2007; Clabo, 2008; El-Nemer, Downe, \& Small, 2006). Qualitative research is appropriate for understanding the experiences of individuals regarding a particular phenomenon (As de Vos, Strydom, Fouche, \& Delport, 2011; Patton, 2002). Hence, the pre-operative experiences of general surgical patients were illuminated during individual interviews.

\section{Setting}

The study was conducted at a tertiary level hospital and a regional hospital (Korle-bu Teaching Hospital [KBTH] and Ridge Hospital respectively) in Accra, the capital of Ghana. The hospitals attract patients from all parts of the country. This could be attributed to the cosmopolitan nature of Accra and the effect of urbanization that attract Ghanaians from other parts of the country.

\section{Population and Sampling}

The study targeted Ghanaian surgical patients who under-went both emergency and planned general surgical procedures such as removal of the breast, appendix, thyroid gland, and repair of hernia and other abdominal surgeries. The surgical procedures have different prognosis and it provided a broader scope of pre-operative experiences of patients. Patients of both sexes were involved in the study and they were from various parts of Ghana such as the South, the middle belt, and the north. Purposive and convenience sampling methods were applied and this ensured the appropriate patients were recruited. The patients involved in the study were from low and average socioeconomic background. The sample involved 13 patients for individual interviews. 


\section{Data Collection Method}

Patients were recruited for individual interviews post-operatively. Recruitment was done 2 to 5 days post-operative when the patient was not in pain. The interviewer observed for signs pain or distress during the interview so that interviews could be re-scheduled in such instances. Individual interviews were conducted at an assigned room on the ward and interview sessions were not interrupted. Interview sessions lasted for 30 to 45 minutes and were recorded and transcribed. A broad initial question: 'Please tell me about your recent surgery; in particular, your experiences before the surgery' elicited the pre-operative experiences of patients and patients responses were further probed to gain a full understanding. In this study pre-operative experience was considered all incidences and care activities from the time decision of surgery was made until surgery was performed. Also, pre-operative anxiety was considered worry or uneasiness associated with an impending surgery. Follow-up interactions on themes emerging from interviews and keeping detailed field notes helped to understand pre-operative experiences of patients.

Interviews were conducted in English, Twi, and Ewe as appropriate. Interviews conducted in English were transcribed verbatim and those conducted in Twi and Ewe were transcribed in English focusing on the meaning of accounts. Transcripts were discussed with peers experienced in Twi and Ewe and with patients and any discrepancies were addressed. The reviews ensured that although transcription in Twi or Ewe, translation and back-translation were not employed in this study, participants' accounts were considered accurate after participants' and expert reviews of transcripts.

\section{Ethics}

The study was approved by the ethics committee at the University of the Western Cape and the Ghana Health Service Ethics Committee. Formal approvals were obtained from the authorities of the two hospitals involved in the study and individual participants gave informed content. Permission was obtained to record interviews and ID codes were used to ensure confidentiality and anonymity (MPi to MP6 - male patients and FPi to FP7 - female patients interviewed).

\section{Rigour}

Rigour or trustworthiness was ensured by employing multiple data collection methods which triangulated or verified patients' accounts. Prolonged field work of more than 6 months also ensured that full understanding of pre-operative experiences was obtained. Member-checking was also employed and it ensured that participants' experiences were truthfully represented and the process also verified interviews that were not conducted in English (Patton, 2002).

\section{Data Analysis}

Transcripts and field notes were read several times to have an in-depth understanding of participants' pre-operative experiences. Data analysis occurred concurrently and principles of content analysis were followed systematically (Graneheim \& Lundman, 2004). When meaning was made out of the data generated, the data was exported into NVivo 9 and the software was used to manage data subsequently. 


\section{Findings}

The findings in relation to the pre-operative experiences and care of patients were themed as reaction to impending surgery and information gap. The two themes have been described with supporting verbatim quotations from patients.

\section{Reaction to Impending Surgery}

This theme describes the reactions of patients to their impending surgery. Sub-themes that emerged included inappropriate disclosure; fear of death; readiness for surgery; and effect of waiting in the theatre.

\section{Inappropriate Disclosure}

Some patients reported that they experienced emotional stress when their initial diagnosis was disclosed. It was realized that women who had breast cancer experienced strong emotional response for the loss of their breast when the need for surgery was disclosed. Some women cried and were depressed when they were told that their breast will be

removed. The women were devastated about the blunt manner in which the diagnosis of breast cancer was disclosed and the need for mastectomy was presented. 'In fact the way and manner the doctor told me that I had breast cancer was shocking; and I couldn't move; I wept. The doctor took my papers, looked through it and then she told me she was going to consult a senior doctor. But before she left, her face was not very good. From the senior doctor, she didn't even sit down and she said 'I am afraid your breast would be cut' In fact, it was only God who saved me; that scared me. I was just crying and talking to myself...' (FP2).

\section{Fear of Death}

This theme describes patients' emotional reactions to impending surgery. The type of emotional reaction exhibited by a patient related to the type of surgery, and previous surgery. Thus, some patients were afraid that they would die during surgery. It was realized that some patients who had emergency surgery and those without previous surgical experience were afraid of surgery. For example $\mathrm{FP}_{5}$ who had an emergency appendicectomy said 'I was afraid because I didn't know how the operation would be since it is my first time'.

However, some patients who had planned surgeries also experienced fear pre-operatively. It was realized that although participants were afraid of the impending surgery, they agreed to undergo the surgery.

'After he (doctor) had given me the date for the surgery, I was frightened and whenever I remembered I will be going to the theatre, I got frightened. So, I was praying that God should take me through and He has taken me through successfully. It was my faith that took me to the theatre because I knew that I had to do it in order to be well' (FP7).

It was also realized that some patients had previous misconceptions about surgery and these influenced their perceptions about the surgical experience. However, they realized that the previous misconceptions were exaggerated.

'I knew that operation was very scary; but I have seen that it is not that scary. People said if you are sent to the operation room, you will see an axe and metal (cutlasses) and other things 
and you will shake and if God's grace is not sufficient on you, you will die. When I came, that thought was on my mind. But I was not afraid because I had faith in God for a successful operation. So I have realized that even though they said it was scary, it was not that scary' (MP2).

\section{Readiness for surgery}

A few of the patients were not afraid or 'did not feel any uneasiness' (FP3). This wasattributed to readiness to cure or remove the diseased body part and health personnel chatting with patient in the theatre.

'I wasn't afraid, because I was also willing to be operated to improve my health, ...I was ready to cure my sickness, because the thing is disturbing me and the best thing is to give my body, so I was ready to do that' (MP2).

'When I was being taken to the theatre, in fact I didn't feel any uneasiness and then even at the theatre, the way they were chatting with me, I wasn't afraid, I was actually relaxed' (FP3).

Also, patients who had previous surgery were not afraid of the impending surgery. For example, FPi said: '.this is my $2^{\text {nd }}$ operation; so going to theatre wasn't new to me'.

Also, a strong faith in God also contributed to the readiness for surgery. Some participant reported that they had strong faith in God and they would have safe surgery. For example, MP6 said: 'because I am a believer, I had faith that I would go and return, so fear was not part of me at all'.

\section{Effect of Waiting in the Theatre}

Also, it was reported that watching other patients being wheeled in and out of the theatre resulted in fear as participants waited for their turn in the theatre. The patients reported that watching the effect of surgery such as shivering on other patients made them afraid. '.when I was waiting in the theatre, I saw someone shivering on the bed after an operation; in fact, I had some fear, the man was shivering severely; if he was brought out before I was sent in, I wouldn't have harboured any fears and I would have been bold to undergo the operation.'(MP4).

Also, some patients were scared because they over-heard some comments from health professionals at the theatre. It was realized that some negative comments made by doctors as they communicated with each other scared the patients before surgery. '.when I was at the theatre, the doctor came and he called another doctor and he said, 'have you seen hers? can you do it?' and he said 'I will try' and I thought eih! You will try! That scared me and in fact, I was serious and I was praying and I told God that 'please I didn't say I needed any I will try doctor; so please God I beg you' later, a specialist came and they took me inside. I didn't see anything' (FPi). 


\section{Information Gap}

It was realized that some patients did not receive adequate information about their surgery and subsequent care. This theme describes the inadequate information experienced by patients about their surgery such as pre-operative care, expectations at the theatre, and undue delays to confirm diagnosis and to perform surgery.

\section{Pre-operative care}

The study realized that surgical patients went through some pre-operative preparation such as laboratory and radiological investigations and other physical preparations on the ward such as shaving and removal of contraindicated items. It was reported that health professionals such as nurses, doctors, and the anaesthetists were involved in pre-operative care. Hence, the anaesthetists assessed patients for planned surgery and declared them fit for surgery.

'.so I saw the doctor and he said: 'go and see the anaesthesia team'; when I went, they also said 'oh my dear you are not fit; you are not strong enough for the surgery so go back to the matron and tell her' (FP2).

However, those for emergency surgeries did not go for assessment at the anaesthetists' clinic.

'.the doctor ...handed me over to some nursing sisters and they took care of me and they said I should carry out some lab tests and $x$-ray. I was then sent to the ward and prepared for the theatre ...' (MP5).

\section{Expectations at the Theatre}

Participants reported that they did not receive adequate information about their impending surgery and what to expect in the theatre. At the tertiary facility which is also a teaching hospital, patients expected information on the presence of students in the theatre. 'they should explain everything to you so that you will be aware before you come; the theatre; when I was going, I thought I was going to meet only my doctors - they were only two; but there were so many doctors there; not knowing, this place is a Teaching hospital so there were nurses, anaesthetist, doctors, medical students; so before they could start; the doctor was explaining some terms to the students' (FP7).

Some participants reported that they watched previous unsuccessful surgical procedures on television and it made them afraid of their impending surgery. Also, some of the patients who had no prior surgical experience were scared of the sophisticated equipment in the operating theatre.

'I didn't think I would live to see a day like this because when I went to the theatre and they were bringing the machines, the knives, and other things, I was watching it; and I was scared. I nearly told them I was going to urinate and I wouldn't have gone back; ... the lights; when the computers were turned on, you hear some sounds; and sometimes when you are watching movies, you will see all those things. I normally thought it was a movie; but when I went, I saw that it was real; and I began to imagine; sometimes after 
the operation, the person will not come back to life; they will be pressing the person and you will see that all the doctors give up; so I began to think of this and something came on my mind that 'that was them; but not you' (MPi).

\section{Undue Delays}

As part of the pre-operative processes, patients with tumours may undergo an initial procedure where a biopsy is taken for histological analysis. The outcome of the analysis informs the type of surgery and subsequent treatment for the patient. Thus, some surgical patients who had planned surgeries reported that they experienced some delay in confirming their diagnosis when biopsies were taken for analysis. '.the doctor removed the lump; then he gave me the specimen and I sent it to the lab); the date that they gave me to come for the results, when I came, it wasn't ready. So, every two weeks I was coming to check. Sometimes when I came some of the lab personnel will just leave the room; then all of a sudden I saw the lump developing again' (FP2).

Also, some patients reported that delay in under-going scheduled surgery was due to industrial action by health professionals, unavailability of anaesthetists, or inadequate facilities such as bed space. Thus, their surgeries were postponed and even after they were admitted, they were asked to go home.

'I was admitted on the ward for the first day and the next day I was sent to the theatre, I spent almost one hour at the theatre and I was sent back to the ward that there were some emergency cases so I should go home, which I didn't understand but maybe it was the will of God so I have to take it like that. I came for the second time and on the third day of admission; I was asked to go home again which I didn't understand' (MP2).

\section{Discussion}

Some of the pre-operative experiences of Ghanaian surgical patients identified in this study were similar to previous studies such as fear of death and inadequate information (Guo et al., 20i2; Rosen et al., 2008) and other findings were specific to the Ghanaian context such as misconceptions about surgery, effect of waiting in the theatre, and faith in God. Ghanaian surgical patients feared for their life pre-operatively due to a number of reasons such as previous misconceptions about surgery. It was realized that the pre- surgery perceptions of patients created unfounded fear that participants attested were exaggerated. Perhaps the perception of fear of death contributed to the observance among some cultures in Ghana where a formal process of celebration is organized for an individual who goes through a successful surgery. In some cases, the celebration is elaborate with food, drinks and merry making and the patient and loved ones wear white garments to show victory over death as in the Ewe culture (the first author's native culture). Other cultures in Ghana such as the Fantes would also go to church and thank God after a successful surgery. This finding demands the need for intensified education about the safety of modern surgery to de-emphasize the fear of death associated with surgery.

Similar to other studies it was realized that patients who did not have previous surgical experience reported more fear than those with previous surgeries (Matthias \& Samarasekera, 
2012; Rosen et al., 2008). It is noted that each surgical process elicits similar repercussions such as bleeding, pain, and infection and a previous surgical exposure may not reduce the risk associated with a current surgery. Thus, patients' lack of fear should not be misconstrued by health professionals to avoid a thorough pre-operative assessment. Also, patients with previous surgeries should be assisted to appreciate the need to be assessed and educated especially if the current surgery is different. Some of the patients who had emergency surgery and had never undergone surgery were afraid because of exposure to previous unsuccessful surgeries they had observed on Television. It is emphasized that with modern technology and effective pre-operative assessment, the incidence of mortality during surgeries is rare. Therefore, patients should not be scared and associate their impending surgeries with what they observe on television since research has shown that mortalities associated with surgery has reduced over the years (Antonacci, Lam, Lavarias, Homel, \& Eavey, 2009; Hutter et al., 2006).

The study realized that participants' personal decision making influenced participants' pre-operative experience. The decision to have a diseased part of the body removed contributed to the lack of fear pre-operatively. Also, environmental factors such as observation of effect of surgery on other post-operative patients in the theatre resulted in fear. It could be inferred that surgical environments where adequate privacy is ensured for pre-operative patients awaiting surgery could prevent pre-operative fear. Adequate privacy could also help minimize communication between health professionals which patients over-heard. An effective communication between patients and health professionals could promote a trusting relationship and enhance the pre-operative experience.

Pre-operative experiences related to professional lapses identified in this study were undue delays to confirm diagnosis and to perform surgery. It is realized that adequate measures to expedite diagnostic measures and perform surgeries as scheduled could enhance pre- operative experiences of surgical patients in Ghana. The emotional changes emanating from these pre-operative experiences could be prevented with appropriate interventions within the context of the study. For example, hospital authorities should take responsibilities for providing the needed facilities and personnel to prevent undue delays pre-operatively. The personnel involved in pre-operative care should undergo regular training and hospitals could develop appropriate guidelines to guide pre-operative care. The findings in this study can be related to previous studies where pre-operative emotional changes such as fear and anxiety resulted in increased post-operative pain (Cohen, Musgrave, Munsell, Mendoza, \& Gips, 2005).

The study also realized that Ghanaian surgical patients go through surgery based on their faith in God. They believed that God would save them from any unfortunate intra- operative incident. The influence of religiosity in the socio-cultural system in Ghana has been explored by sociologists and it is realized that Ghanaians believe in God and associate ill-health and other misfortunes to evil forces. Thus, the belief that God would grant them a successful surgery pre-supposes that the patients believed that God's power would deliver them from any complications. It could also be inferred from this finding that some patients did not trust the health system or the competence of the health team but rather, God; to see them through a successful surgery (Nukunya, 2003; Sackey, 2002). Thus, health professionals should acknowledge the religious aspect of the Ghanaian culture and assist patients to meet their religious needs as appropriate before patient goes to the theatre. Studies have reported contrary 
findings about effect of prayer on surgery among different cultures (Ikedo, Gangahar, Quader, \& Smith, 2007).

The study also realized that the process of disclosure of primary diagnosis that warranted surgery was sometimes not done with sensitivity. Women with breast cancer who had mastectomy reported that some doctors were not sensitive in disclosing the initial diagnosis. It is reported that some women delay mastectomy surgeries due to the potential effect on their body image, marriage, and sexuality (Aziato, 2006). Thus, surgeries with potential effect on the body image should have particular pre-operative attention in preparing patients and their relatives as necessary. Strong emotional reaction reported for women scheduled for breast surgery could be due to the poor prognosis of late breast cancer common among Ghanaian women (Clegg-Lamptey \& Hodasi, 2007). Perhaps health personnel should be trained to acquire skills in disclosing and counselling patients with sensitive diagnosis.

In congruence with previous studies, Ghanaian pre-operative patients reported that they were not provided with adequate information pre-operatively (Chan, Kan, Lee, Chan, \& Lam, 20ii). Patients scheduled for emergency surgery exhibited lack of knowledge on what to expect in the theatre (Rosen et al., 2008). Although patients for planned surgeries who attended the anaesthetists' clinic were provided with information about their surgeries, it was perceived that the information provided needed to be reinforced and expanded. The pre-operative information could include a section on the set-up of the theatre and a video of the equipment and procedures in the theatre. The tertiary facility is a teaching hospital and various categories of students have their clinical experiences in the hospital. Hence, patients should be informed about the involvement of students in their care and their consent should be obtained in such cases.

\section{Implications of the Study}

The contextual findings illuminated imply the need for intensified education of Ghanaians to limit the fear of death associated with surgery. Also, effective pre-operative assessment and maintaining high intra-operative standards are necessary to ensure safe surgery. It is also important that patients scheduled for surgery are not made to wait in the theatre; they should be on the ward and sent to the theatre according to the operation list. In cases where patients have to wait in the theatre, privacy should be provided so that they do not see other freshly operated patients wheeled out of the operating room.

Health professionals in Ghana should appreciate the role of religiosity among surgical patients and allow patients and relatives to observe their religious needs as appropriate. The faith in God reported by patients also implies health professionals should perform their roles diligently during surgery so that patients' expectations of surgery are met and this could perpetuate their continued faith in God.

\section{Limitations of study}

The study involved only Christians and application of findings among other religious contexts may be inappropriate. Also, like other qualitative studies, the small sample size involved does not permit generalization to all Ghanaian surgical patients. Thus, future studies could adopt 
quantitative measures to confirm applicability of findings to other contexts in Ghana. Also, the extent of fear or anxiety could be measured to enhance the discourse in this area of research especially considering the different prognosis of various surgical procedures as in this study. Future studies could also investigate the impact of socio-economic levels of patients on pre-operative anxiety especially for patients without health insurance.

\section{Conclusion}

The pre-operative experiences of patients vary depending on factors such as the urgency of surgery, type of surgery, and existing medical conditions. Effective pre-operative preparation is known to enhance post-operative pain management and recovery. Health professionals need to be cognizant of the contextual factors that influence patients' pre- operative experiences and give context appropriate care. Also, patients and their relatives should be assisted to appreciate the importance of pre-operative preparation so that they would cooperate in the care at this phase. The safety of modern surgery should be highlighted to de-emphasize the fear of death associated with surgery within the Ghanaian context. The need for intensified pre-operative education of patients and their relatives is highlighted in this study.

\section{References}

Antonacci, A. C., Lam, S., Lavarias, V., Homel, P., \& Eavey, R. A. (2009). A report card system using error profile analysis and concurrent morbidity and mortality review: Surgical outcome analysis, part II. Journal of Surgical Research, 153(1), 95-104. doi: 10.1016/j.jss.2008.02.051

Arber, A. (2007). "Pain talk" in hospice and palliative care team meetings: An ethnography. International Journal of Nursing Studies, 44(6), 916-926. doi: 10.1016/j.ijnurstu.2006.04.002

As de Vos, Strydom, H., Fouche, C. B., \& Delport, C. S. L. (2011). Research at grass roots: for the social sciences and human service professions (4th ed.). Pretoria: Van Schaik Publishers.

Asilioglu, K., \& Celik, S. S. (2004). The effect of preoperative education on anxiety of open cardiac surgery patients. Patient Education and Counseling, 53(1), 65-70. doi: 10.1016/So738- 3991(03)o0117-4

Aziato, L. (2006). Post-mastectomy experiences of women with breast cancer in the Accra metropolis. West African Journal of Nursing, 20(1^51-57.

Badoe, E. A. (2009). A brief history of surgery. In Badoe, E. A., Archampong, E. Q., \& da Rocha- Afodu , J. T. (Eds.), Principles and practice of surgery including pathology in the tropics (pp. 1-11). Tema: Ghana Publishing Corp.

Brady, M. C., Kinn, S., Stuart, P., \& Ness, V. (2010). Preoperative fasting for adults to prevent perioperative complications. In Cochrane Database of Systematic Reviews: John Wiley \& Sons, Ltd. doi: 10.1002/14651858^004423. 
Chan, Z., Kan, C., Lee, P., Chan, I., \& Lam, J. (2011). A systematic review of qualitative studies: Patients' experiences of preoperative communication. Journal of Clinical Nursing, 21(5-6), 812-824. doi:10.1111/j.1365-2702.2011.03942.x

Clabo, L. M. L. (2008). An ethnography of pain assessment and the role of social context on two postoperative units. Journal of Advanced Nursing, 61(5), 531-539.

Clegg-Lamptey, J. N. A., \& Hodasi, W. M. (2005). An audit of aspects of informed consent and pain relief in general surgical units of Korle-Bu Teaching Hospital. Ghana Medical Journal, 39(2), 63-67. PMCID: PMC1790812.

Clegg-Lamptey, J. N. A., \& Hodasi, W. M. (2007). A study of breast cancer in Korle-Bu Teaching Hospital: Assessing the impact of health education. Ghana Medical Journal, 41(2), 72-77. PMCID: PMC2002569.

Cohen, M. Z., Musgrave, C. F., Munsell, M. F., Mendoza, T. R., \& Gips, M. (2005). The cancer pain experience of Israeli and American patients 65 years and older. Journal of Pain and Symptom Management, 30(3), 254-263. doi: 10.1016/j.jpainsymman.2005.03.011

El-Nemer, A., Downe, S., \& Small, N. (2006). 'She would help me from the heart': An ethnography of Egyptian women in labour. Social Science \& Medicine, 62, 81-92. PMID: 16005133

Graneheim, U. H., \& Lundman, B. (2004). Qualitative content analysis in nursing research: concepts, procedures and measures to achieve trustworthiness. Nurse Education Today, 24(2), io5-ii2. doi: io.ioi6/j.nedt.2003.io.ooi

Guo, P., East, L., \& Arthur, A. (20i2). A preoperative education intervention to reduce anxiety and improve recovery among Chinese cardiac patients: A randomized controlled trial. International Journal of Nursing Studies, 49(2), i29-i37. doi: io.ioi6/j.ijnurstu.20ii.o8.o08

Hutter, M. M., Rowell, K. S., Devaney, L. A., Sokal, S. M., Warshaw, A. L., Abbott, W. M., et al. (2006). Identification of surgical complications and deaths: An assessment of the traditional surgical morbidity and mortality conference compared with the American College of Surgeons-National Surgical Quality Improvement Program. Journal of the American College of Surgeons, 203(5), 6i8-624. doi: io.ioi6/j.jamcollsurg.2006.07.oio

Ikedo, F., Gangahar, D. M., Quader, M. A., \& Smith, L. M. (2007). The effects of prayer, relaxation technique during general anesthesia on recovery outcomes following cardiac surgery. Complementary Therapies in Clinical Practice, 13(2), 85-94. doi: io.ioi6/j.ctcp.2006.io.004 
Matthias, A. T., \& Samarasekera, D. N. (20i2). Preoperative anxiety in surgical patients experience of a single unit. Acta Anesthesiologica Taiwanica, 5o(i), 3-6. doi: io.ioi6/j.aat.20i2.02.004

Mullen, P., van den Borne, B., \& Breemhaar, B. (2000). Implementing a surgery patient education program as a routine practice: A study conducted in two Dutch hospitals. Patient Education and Counseling, 41(2), 223-234. doi: io.ioi6/So738-399i(oo)ooi40-3

Nukunya, G. K. (2003). Tradition and change: an introduction to sociology (2nd ed.). Accra: Ghana Universities Press. Patton, M. Q. (2002). Qualitative research and evaluation methods. London: Sage Publications Inc.

Qu, S., Sherwood, G. D., McNeill, J. A., \& Zheng, L. (2008). Postoperative pain management outcome in Chinese inpatients. Western Journal of Nursing Research, 30(8), 975-990. doi: io.ii77/oi939459083i9576

Rosen, S., Svensson, M., \& Nilsson, U. (2008). Calm or not calm: The question of anxiety in the perianesthesia patient. Journal of PeriAnesthesia Nursing, 23(4), 237-246. doi: io.ioi6/j.jopan.2008.05.002

Sackey, B. M. (2002). Faith healing and women's reproductive health: Institute of African Studies. Sociocultural dimension of reproductive health and human development. Research Review New series: Univ. of Ghana, i8(i).

Sjoling, M., Nordahl, G., Olofsson, N., \& Asplund, K. (2003). The impact of preoperative information on state anxiety, postoperative pain and satisfaction with pain management. Patient Education and Counseling, 51(2), i69-i76. doi: io.ioi6/So738-399i(02)ooigi-

X Solca, M. (2006). Evidence-based preoperative evaluation. Best Practice \& Research Clinical Anaesthesiology, 20(2), 23^236. doi: io.ioi6Zj.bpa.2005.io.003

Sereide, E., \& Ljungqvist, O. (2006). Modern preoperative fasting guidelines: A summary of the present recommendations and remaining questions. Best Practice \& Research Clinical Anaesthesiology, 20(3), 483-49L doi: io.ioi6/j.bpa.2006.03.002

Stepanovic, S., Vukovic, D., Bedora-Faure, M., K'ouas, G., Djukic, S., Svabic-Vlahovic, M., et al. (2007). Surgical wound infection associated with Psychrobacter phenylpyruvicus-like organism. Diagnostic Microbiology and Infectious Disease, 57(2), 2i7-2i9. doi: io.ioi6/j.diagmicrobio.2006.08.002

Hutter, M. M., Rowell, K. S., Devaney, L. A., Sokal, S. M., Warshaw, A. L., Abbott, W. M., et al. (2006). Identification of Surgical Complications and Deaths: An Assessment of the Traditional Surgical Morbidity and Mortality Conference Compared with the American 
College of Surgeons-National Surgical Quality Improvement Program. Journal of the American College of Surgeons, 203(5), 6i8-624. 